Universidade de São Paulo

Museu de Zoologia

Elias da Costa Araujo Junior

\title{
Taxonomia e análise cladística de Theages Walker, 1855 (Lepidoptera, Erebidae, Arctiinae, Arctiini, Ctenuchina)
}

São Paulo 
Universidade de São Paulo

Museu de Zoologia

Programa de Pós-graduação em Sistemática, Taxonomia Animal e Biodiversidade

Taxonomia e análise cladística de Theages Walker, 1855 (Lepidoptera, Erebidae, Arctiinae, Arctiini, Ctenuchina)

Dissertação apresentada ao Programa de PósGraduação em Sistemática, Taxonomia Animal e Biodiversidade do Museu de Zoologia da Universidade de São Paulo como pré-requisito para obtenção do título de Mestre.

Orientador: Prof. Dr. Marcelo Duarte da Silva Co-orientadora: Prof. Dra. Lívia Rodrigues Pinheiro

São Paulo 
Araujo Junior, Elias da Costa

Taxonomia e análise cladística de Theages Walker, 1855 (Lepidoptera, Erebidae, Arctiinae, Arctiini, Ctenuchina)./ Elias da Costa Araujo Junior; orientador Marcelo Duarte da Silva e coorientadora Livia Rodrigues Pinheiro. São Paulo, 2018.

$131 \mathrm{p}$.

Dissertação (Mestrado) - Programa de Pós-Graduação em Sistemática, Taxonomia e Biodiversidade, Museu de Zoologia, Universidade de São Paulo, 2018.

Versão original

\section{Comissão Julgadora}

Prof. Dr.

Julgamento:

Prof. Dr.

Julgamento:

Prof. Dr.

Julgamento:
Instituição:

Assinatura:

Instituição:

Assinatura:

Instituição:

Assinatura: 


\section{Resumo}

Theages Walker, 1855 é um gênero estritamente Neotropical constituído por 12 espécies bastante similares entre si quanto aos caracteres cromáticos, revelando-se uma considerável confusão taxonômica e nomenclatural. No presente trabalho, sob uma pespectiva de morfologia comparada, buscou-se reconhecer e elucidar o número de táxons nominais pertencentes ao gênero, bem como propor uma hipótese de relacionamento evolutivo entre eles. A análise cladística contou com 17 terminais (11 do grupo interno e seis do grupo externo) e 41 caracteres morfológicos. A busca exaustiva de pesos iguais resultou em uma única árvore mais parcimoniosa (l=142) e a de busca por pesagem implícita também gerou uma única árvore mais parcimoniosa (l=143). O gênero teve sua monofilia sustentada por oito sinapomorfias, sendo cinco únicas e três homoplásticas. Foram propostos ainda dois complexos de espécies para o gênero: (T. xanthura (T. leucophaea, T albidius)) e (T. pinasi (T. griseatum, T. hoffmanni)) (T. occultus (T. flavicaput, Theages sp. nov.))). Adicionalmente, com base na topologia obtida são fornecidos subsídios para o entendimento da evolução dos caracteres sexuais secundários encontrados no gênero. A revisão taxonômica, por sua vez, permitiu o reconhecimento de 11 espécies válidas, com a inclusão de uma espécie nova para a Colômbia e Equador. Uma sinonímia também foi proposta para o gênero: T. bricenoi torna-se sinônimo júnior subjetivo de $T$. flavicaput. Todas as espécies são redescritas, ilustradas e tiveram suas distribuições geográficas mapeadas e discutidas. Uma chave de identificação também é fornecida. 


\begin{abstract}
Thaeges Walker, 1855 is a strictly Neotropical genus composed of 12 species very similar amongst themselves regarding chromatic characters, thus revealing a considerable taxonomic and nomenclatural confusion. In the present study, under the perspective of comparative morphology, the recognition and elucidation of the number of nominal taxa belonging to this genus was aimed, as well as proposing an evolutionary relationship hypothesis between them. The cladistic analysis comprised 17 terminals (11 from the ingroup and six from the outgroup), and 41 morphological characters. The exhaustive search for equal weights resulted in a single most parsimonious tree $(\mathrm{I}=142)$ and the implicit weight search also yielded a single most parsimonious tree (I=143). The genus' monophyly was well supported by eight synapomorphies, five being unique and three homoplastic. In addition, two species complexes were proposed for the genus: (T. xanthura (T. leucophaea, T albidius)) and (T. pinasi (T. griseatum, T. hoffmanni)) (T. occultus (T. flavicaput, Theages sp. nov.))). Furthermore, based on the topology obtained, subsidies for the understanding of the evolution of the male secondary sexual characters found in the genus were provided. On the other hand, the taxonomic revision enabled the recognition of 11 valid species with the inclusion of a new species for both Colombia and Ecuador. Additionally, a synonym was also proposed to the genus: T. bricenoi becomes a junior subjective synonym of $T$. flavicaput. All species were redescribed, illustrated, and their geographical distributions mapped and discussed. An identification key was also provided.
\end{abstract}




\section{INTRODUÇÃO}

\subsection{Contexto taxonômico}

O presente trabalho tem como alvo o gênero Neotropical Theages Walker, 1855 que é constituído atualmente por 12 espécies de mariposas com hábito noturno.

A classificação atual de Theages é fornecida a seguir, com informações sobre cada grande arranjo taxonômico em que Theages faz parte:

Ordem Lepidoptera

Superfamília Noctuoidea

Família Erebidae

Subfamília Arctiinae

Tribo Arctiini

Subtribo Ctenuchina

\subsection{Ordem Lepidoptera}

Lepidoptera é a segunda ordem mais diversa de insetos, sendo representada pelas borboletas e mariposas, reconhecidas pelo corpo coberto por escamas (Duarte et al., 2012). São conhecidas cerca de 160.000 espécies no mundo, distribuídas em uma ampla variedade de habitats e regiões (Goldstein, 2017; Mitter et al., 2017). Apesar de Lepidoptera constituir um grupo bem estudado, principalmente com relação às borboletas (Gaston, 1991), as estimativas sobre a sua diversidade, geralmente, expressam números bastante contrastantes, desde 146.000 até 500.000 espécies (Heppner, 1991; Kristensen et al., 2007; Piovesan et al., 2014). Entretanto, mesmo imersa em um cenário com tantas controvérsias, estima-se que o número de espécies da ordem seja excedido apenas pela riqueza de himenópteros, coleópteros e angiospermas (Kristensen et al., 2007; Forbes et al., 2018).

Os lepidópteros desempenham um papel chave nos ecossistemas terrestres, participando de várias interações ecológicas, tais como a polinização e herbivoria (Regier et al., 2009). Além disso, as larvas geralmente são elos importantes em teias tróficas e possuem estreita relação com as plantas hospedeiras (DeVries, 1986; Diniz et al., 1999; Grimaldi \& Engel, 2005), uma vez que as fêmeas de algumas espécies depositam seus ovos em plantas especifícas e as larvas são, em sua maioria, fitófagas 
(Ehrlich \& Raven, 1965). Dessa forma, não é coincidência que a maior irradiação de Lepidoptera tenha ocorrido juntamente com as angiospermas (Powell et al., 1998). Neste cenário, em uma escala geológica, a dominância dos lepidópteros parece ser relativamente recente (Kristensen, 1984). Existe uma divergência com relação ao surgimento dos lepidópteros, com algumas evidências apontando os primeiros registros confiáveis de fósseis da ordem para o Cretáceo Inferior (Whalley, 1978) e outras para o final do Terciário (Eldijk et. al., 2018), embora muitos dos fósseis encontrados nos estratos anteriores ao Terciário e, previamente inseridos em Lepidoptera, tenham sido realocados em outras ordens posteriormente (Crowson et al., 1967). Apesar disso, estima-se que a ordem constitui a maior radiação de insetos fitófagos da Terra (Scoble, 1992).

Essa diversidade expressiva, combinada com várias outras características, tais como a ampla distribuição geográfica e padrões de coloração atraentes, resultou em uma quantidade relevante de estudos focados na taxonomia e sistemática da ordem (Kristensen et al., 2007), sendo sua monofilia fortemente suportada por mais de vinte sinapomorfias morfológicas (Heppner, 1998; Kristensen \& Skalski, 1998; Kristensen et al., 2007). O seu posicionamento dentro de Insecta, por sua vez, é similarmente bem estabelecido, tendo Trichoptera como grupo-irmão, ambas constituindo o clado denominado Amphiesmenoptera (Ross, 1967; Kristensen, 1984; Chalwatzis et al., 1996; Kristensen, 1997; Kristensen, 1999; Kjer et al., 2001; Beutel \& Pohl, 2006).

Adicionalmente, muitos grupos de lepidópteros se tornaram indicadores importantes na Biologia da Conservação e, devido aos padrões de coloração, são comumente utilizados como espécies bandeira e espécies guarda-chuva em programas para a manutenção de áreas naturais remanescentes (New, 1997).

\subsection{Superfamília Noctuoidea}

Noctuoidea é a superfamília mais diversa de Lepidoptera, compreendendo aproximadamente 70.000 espécies catalogadas (Kitching \& Rawlins, 1998), com o número de gêneros variando entre 4.200 e 6.500 (Poole, 1989; Kitching \& Rawlins, 1998; Zahiri et al., 2011). O número de famílias reconhecidas, por sua vez, também pode variar dependendo da fonte consultada (Zahiri et al., 2010). Não obstante, é provável que exista um número significativo de espécies não descritas, 
particularmente da região tropical, além de muitos conceitos genéricos que necessitam de extensa revisão e redefinição de seus limites (Zahiri et al., 2011; Holloway, 2011).

Tradicionalmente, quatro termos relativos à venação alar são comumente utilizados em discussões sobre a sistemática da superfamília. Com base no posicionamento da veia $\mathrm{M}_{2}$ das asas anteriores, Noctuoidea é dividida em trífideos, com $\mathrm{M}_{2}$ surgindo no meio da célula discal e conferindo à veia cubital a aparência de três ramificações; e quadrifídeos, com $\mathrm{M}_{2}$ apresentando origem próxima à $\mathrm{M}_{3} \mathrm{e}$ conferindo à veia cubital a aparência de quatro ramificações (Lafontaine \& Fibiger, 2006). Estes últimos são ainda subdivididos em dois outros grupos com base na venação alar das asas posteriores: trifíneos, com $\mathrm{M}_{2}$ é geralmente vestigial ou ausente e conferindo à veia cubital a aparência de três ramificações; quadrifíneos, com $\mathrm{M}_{2}$ desenvolvida e surgindo no terço posterior da célula discal, geralmente com origem adjacente à $\mathrm{M}_{3}$ em muitas subfamílias, conferindo à veia cubital a aparência de quatro ramificações (Lafontaine \& Fibiger, 2006; Zahiri et al., 2011).

Do total de gêneros inseridos em Noctuoidea, cerca de 500 apresentam espécies com notável importância econômica, pois larvas e adultos podem atuar como pragas em cultivos agrícolas (Bänziger 1982; Kitching, 1984; Zhang, 1994; Mitchell et al., 2006). De acordo com Zahiri et al. (2010), a construção de uma classificação estável, assim como a compreensão das relações filogenéticas dessas mariposas, é um passo de grande importância para estudos futuros focados na bionomia de tais insetos. Tal avanço no conhecimento bionômico poderá abrir portas também para o estudo de características pouco exploradas na literatura ou mesmo ainda desconhecidas que contribuirão para resoluções mais robustas da sistemática do grupo.

A despeito disso, embora os trabalhos de classificação de Lepidoptera tenham sido derivados principalmente dos caracteres de adultos, alguns estudos clássicos com imaturos de representantes de Noctuoidea apontaram que os caracteres larvais poderiam ser úteis não somente na identificação dos táxons, mas também na classificação do grupo (Forbes, 1910; Fracker, 1915; Mosher, 1916; Ripley, 1923; Crumb, 1934; Dethier, 1941). A proposta de classificação de Noctuoidea sugerida por Kitching \& Rawlins (1998), por exemplo, foi a primeira a fazer uso extensivo de caracteres tanto de adultos quanto dos estágios imaturos, fornecendo diversas 
contribuições para classificação do grupo, até então pouco investigada desde o catálogo de Hampson (1898-1913).

Historicamente, Noctuoidea ainda constitui um grupo problemático tanto do ponto de vista taxonômico quanto sistemático, com uma fraca compreensão acerca dos limites e relações evolutivas de seus gêneros e espécies (Mitchell et al., 2000; Zahiri et al., 2010). Nesse contexto, sua classificação tem sido altamente instável, com diferentes sistemas de classificação usados por autores distintos. Além disso, algumas das classificações usadas ao longo do tempo foram baseadas em semelhanças superficiais (Kitching, 1984; Zahiri et al., 2011). Kitching (1984), por exemplo, realizou uma revisão histórica da classificação de alguns grupos alocados na superfamília, fornecendo uma discussão acerca dos caracteres que geralmente são empregados na taxonomia do grupo.

Apesar da monofilia de Noctuoidea ser fortemente sustentada pela presença de um órgão timpânico metatorácico (Miller, 1991) e estruturas abdominais associadas (Kitching \& Rawlings, 1998; Mitchell et al., 2006), progressos na resolução de muitas das incertezas taxonômicas e filogenéticas abarcadas na subfamília se tornaram evidentes apenas nas últimas décadas (Miller, 1991; Weller et al., 1994; Mitchell et al., 1997; Kitching \& Rawlins, 1998; Fibiger \& Lafontaine, 2005; Lafontaine \& Fibiger, 2006; Mitchell et al., 2006; Zahiri et al., 2010; Zahiri et al., 2011; Regier et al. 2016). Miller (1991) reconheceu a presença do órgão timpânico metatorácico como a única sinapomorfia de Noctuoidea, uma estrutura auditiva altamente especializada, capaz de conferir tanto a detecção de sinais de ecolocalização, e que provavelmente pode estar envolvida na recepção de sinais de acasalamento (Kitching \& Rawlins, 1998).

O trabalho de Miller (1991) reconheceu ainda sete famílias dentro de Noctuoidea: Oenosandridae, Doidae, Notodontidae, Lymantriidae, Arctiidae, Aganaidae e Noctuidae. Scoble (1992) considerou Aganainae como uma subfamília de Noctuidae, reconhecendo, portanto, apenas seis famílias. Posteriormente, novas mudanças adentraram na história taxonômica do grupo, com o reconhecimento de três principais linhagens dentro da superfamília: Oenosandridae, Doidae + Notodontidae, e as famílias Arctiidae, Lymantriidae, Noctuidae, Nolidae e Pantheidae (Kitching \& Rawlins, 1998). 
Valiosas contribuições para a filogenia e para a revisão da classificação de Noctuoidea foram fornecidas por Fibiger \& Lafontaine (2005). Tais autores redescreveram e discutiram alguns dos caracteres inadequadamente descritos e, portanto, mal compreendidos, em estudos prévios focados na sistemática do grupo. Adicionalmente, realizaram diversas mudanças nomenclaturais e reconheceram dez famílias em Noctuoidea: Oenosandridae, Doidae, Notodontidae, Micronoctuidae, Nolidae, Strepsimanidae, Arctiidae, Lymantriidae, Erebidae e Noctuidae. Todavia, em uma proposta subsequente, os mesmos autores rebaixaram as cinco últimas ao status de subfamília, todas inseridas em Noctuidae (Lafontaine \& Fibiger, 2006), baseados na presença de um esclerito timpânico e na venação quadrifídea das asas anteriores.

Mitchell et al. (2006), por outro lado, em uma hipótese baseada em dados moleculares, apontaram a parafilia de Noctuidae sensu Lafontaine \& Fibiger (2006). Aqueles autores consideraram apenas as subfamílias trifíneas como pertencentes ao conceito monofilético de Noctuidae e, portanto, elevaram as quadrifíneas Arctiinae e Lymantridae ao status de família. Posteriormente, Zahiri et al. (2010) forneceram outra hipótese filogenética da superfamília baseada em dados moleculares, sugerindo seis famílias como clados fortemente suportados: Oenosandridae, Notodontidae, Erebidae, Euteliidae, Nolidae e Noctuidae. Dessa forma, os Noctuidae quadrifíneos (Aganainae, Calpinae e Catocalinae), assim como os Arctiidae e Lymantridae, foram considerados como subfamílias alocadas em Erebidae.

Historicamente, embora Arctiidae tenha sido considerada como família de Noctuoidea, optou-se por seguir no presente estudo a classificação sugerida por Zahiri et al., (2010), apesar de ainda haver muito a esclarecer sobre as relações evolutivas dos Noctuoidea. O suporte obtido no referido trabalho tem sido considerado robusto para a adoção geral, sendo prontamente adotado por Van Nieukerken et al., (2011) e por Regier et al. (2016). Este último trabalho baseado em dados moleculares e abarcando todas as famílias de Noctuoidea, bem como a maioria das subfamílias atualmente reconhecidas, também encontrou forte suporte para o sistema de seis famílias sensu Zahiri et al., (2010). Desse modo, Arctiidae será aqui tratada como subfamília de Erebidae. 


\subsection{Família Erebidae}

Erebidae é uma família que se enquadra no arranjo dos quadrifídeos e, dentro destes, nos quadrifíneos, sendo considerada uma das mais diversas de Lepidoptera, com cerca de 24.600 espécies descritas (Van Nieukerken et al., 2011). No entanto, assim como a ausência de uma compreensão clara sobre o número de espécies descritas em Noctuoidea, o número de táxons descritos em Erebidae é ainda mais incerto. Apesar dos avanços nos últimos anos acerca de algumas das incertezas taxonômicas imersas na família, é notório que sua classificação interna ainda requer resoluções mais robustas (Zahiri et al., 2011). Essa conjuntura é marcada, principalmente, pela fraca delineação da família, com poucos trabalhos realizados em tal perspectiva (Fibiger \& Lafontaine, 2005; Mitchell et al., 2006; Regier et al., 2009; Mutanen et al., 2010; Zahiri et al., 2010; Zahiri et al., 2011).

Como de praxe dentro de Noctuoidea, Erebidae também apresenta um histórico problemático, tendo sido desconsiderada por Hampson (1913). Erebidae foi, contudo, revalidada como família no trabalho de Fibiger \& Lafontaine (2005) e considerada por eles como próxima de Arctiidae, Lymantriidae e Nolidae. Tais autores incluíram 17 subfamílias em Erebidae, englobando a revalidação de seis subfamílias: Rivulinae, Boletobiinae, Hypenodinae, Phytometrinae, Scolecocampinae e Erebinae. Apesar da família ter sido diagnosticada com a combinação de vários caracteres morfológicos, seu tratamento como família foi prontamente questionado e, portanto, permaneceu difuso, sendo seu status de família novamente desconsiderado no trabalho de Lafontaine \& Fibiger (2006).

Todavia, Zahiri et al. (2010) recuperam a monofilia de Erebidae, sendo a mesma corroborada novamente em uma hipótese para a compreensão das relações internas do grupo lançada por Zahiri et al. (2011), sendo a mesma baseada exclusivamente em dados moleculares e abarcando todas as subfamílias, assim como uma quantidade expressiva de tribos e subtribos. Apesar de tais autores concordarem com o restabelecimento do grupo como família conforme havia sido sugerido por Fibiger \& Lafontaine (2005), o seu conceito como grupo natural foi reconfigurado e incluiu 18 subfamílias, das quais cinco previamente tratadas como famílias: Arctiidae, Lymantriidae, Micronoctuidae, Herminiidae e Aganaidae. Esse novo arranjo também foi seguido por Regier et al. (2016), que investigaram as relações entre 13 das 18 subfamílias atualmente alocadas em Erebidae e conseguiram um suporte maior do que 
aqueles obtidos nas duas propostas moleculares supracitadas (Zahiri et al., 2010, 2011).

Apesar de tais esforços, a família Erebidae necessita de uma melhor delineação, visto que seu histórico bastante confuso é, em parte, proveniente da ausência de uma definição clara e baseada em caracteres exclusivos. Os adultos e larvas das espécies de Erebidae, em particular, exibem uma diversidade expressiva quanto à coloração, tamanho, comportamento e ecologia (Kitching \& Rawlins, 1998). A apreciável diversidade morfológica, por exemplo, é mencionada por Zahiri et al. (2011) como uma problemática na determinação das relações filogenéticas dentro do grupo, tendo em vista a dificuldade em se estabelecer hipóteses de homologias e, consequentemente, na codificação correta dos caracteres.

\subsection{Subfamília Arctiinae}

Arctiinae tem distribuição cosmopolita e compreende mariposas com corpo robusto, sendo principalmente noturnas, porém com alguns representantes crepusculares ou diurnos (Beccacece et al., 2012). A subfamília constitui um grupo bastante diverso, com aproximadamente 11.000 espécies conhecidas e com grande representatividade na região neotropical (Zaspel et al., 2014; Zenker et al., 2016). Entretanto, o grupo provavelmente sofreu uma superestimativa de gêneros e espécies, em virtude da existência de sinônimos e incertezas taxonômicas (Lamas \& Grados, 1996; Grados et al., 2013). Além disso, provavelmente existe um número elevado de táxons que ainda não foram descritos e pouco se conhece sobre a distribuição geográfica da maioria das espécies do grupo, com diversos casos em que apenas a localidade-tipo é conhecida (Grados et al., 2013), tornando escasso o conhecimento dos seus padrões biogeográficos (Zenker et al., 2016).

Isto posto, é evidente que a classificação de Arctiinae ainda não é clara, com algumas controvérsias quanto ao relacionamento de seus táxons (Grados, 2002). A história taxonômica da subfamília é também bastante atribulada porque muitas espécies de linhagens distintas apresentam semelhanças superficiais entre elas (Weller et al., 2009), refletindo em agrupamentos não naturais. Adicionalmente, a subfamília foi pouco investigada sob o ponto de vista taxonômico e filogenético. A hipótese de monofilia da subfamília Arctiinae, contudo, tem sido sustentada por diversos 
caracteres, como a presença de tímbalos metatorácicos no catepisterno; segmentos A3-A6 das larvas com cerda L3 bisetosa; bolsa IV do tímpano aberta; tímpano com carena contratimpânica pré-espiracular e presença de um par de glândulas dorsais de feromônios associadas às papilas anais (Jacobson \& Weller, 2002). Essa última característica foi mencionada por diversos autores (Bendib \& Minet, 1998; Kitching \& Rawlins, 1998), podendo ser longas, ramificadas ou não ramificadas, e, provavelmente, retraídas pelos músculos longitudinais (Kitching \& Rawlins, 1998).

No trabalho de Zahiri et al. (2011), a subfamília Arctiinae foi considerada grupo-irmão de Aganainae e Herminiinae e, de acordo com a classificação atual, é constituída pelas tribos Arctiini, Amerilini, Lithosiini e Syntomini. Assim como os Aganainae, os arctiines adultos geralmente exibem padrões de coloração visualmente impressionantes, com diversas espécies caracterizadas por suas colorações aposemáticas e muitos casos (especialmente em Syntomini e Arctiini: Ctenuchina) plausíveis a mimetismos extremamente semelhantes de várias espécies de Hymenoptera. Além disso, tanto os imaturos quanto os adultos da subfamília podem utilizar diversos compostos secundários, tais como polifenóis, sesquiterpenos, pirrolizizidínicos, glicosídeos e cardenolídeos que são sequestrados de plantas hospedeiras (Weller et al., 1999).

\subsection{Tribo Arctiini}

Arctiini constitui um grupo bastante diverso, com mais de 4.760 espécies reconhecidas (Heppner, 1991). De acordo Kitching \& Rawlings (1998) a monofilia do grupo é sustentada pela presença de retináculo alongado nos machos e pelos larvópodos com ganchos heteróideos. Jacobson \& Weller (2002) mencionaram cinco sinapomorfias para o grupo: i) ocelos com um anel melanizado ao redor da lente; ii) glândulas de feromônio dorsais das fêmeas divididas e com aspecto digitiforme; iii) apódemas esternais do segmento abdominal II fortemente unidos ao esterno; iv) probóscide não alcançando a região do posterior do tórax, portanto mais longa que a cabeça e v) larvópodos com ganchos heteróideos, única sinapomorfia reconhecida por autores prévios.

Atualmente, Arctiini é dividida nas subtribos Pericopina, Arctiina, Ctenuchina, Euchromiina, Calimorphina e Phaegopterina (Zaspel et al., 2014). 
Segundo Pinheiro \& Duarte (2013), Ctenuchina e Euchromiina são grupos filogeneticamente próximos e compreendem cerca de 3.000 espécies conhecidas, ocorrendo desde a Argentina até o sul dos Estados Unidos. Ainda de acordo com esses autores, são raros os trabalhos de revisão e notas com informações morfológicas na literatura sobre estes grupos.

\subsection{Subtribo Ctenuchina}

A subtribo Ctenuchina é predominantemente neotropical e compreende cerca de 95 gêneros e mais de 800 espécies descritas (Weller et al., 2009). Constitui um grupo taxonomicamente problemático (Simmons e Weller, 2001), sendo tratado historicamente como tribo, subfamília e até mesmo como família por alguns autores (Hampson 1898; Zerny 1912; Draudt 1917). O primeiro tratamento próximo ao conceito moderno do grupo foi o de Lafontaine \& Schmidt (2010) que consideraram tanto Euchromiina quanto Ctenuchina como tribos alocadas em Arctiini.

\subsection{Gênero Theages}

Theages Walker, 1855 está atualmente inserido em Ctenuchina (Zenker et al., 2016) e apresenta características marcantes, pois é o único gênero de Arctiinae com órgãos androconiais odoríferos dos machos na região dorsal do abdome em sulcos transversos, somente visíveis quando o abdome é fortemente comprimido. Além disso, os machos apresentam o nono tergito pouco esclerotizado em contraste com as valvas bem desenvolvidas (Travassos, 1962, 1964). O gênero foi estabelecido com a inclusão de duas espécies: T. leucophaea Walker, 1855 e T. quadricolor Walker, 1855. Inicialmente, o autor não designou a espécie-tipo, sendo T. leucophaea definida posteriormente como tipo por Kirby em 1892.

Assim como diversos gêneros de Arctiinae, Theages apresenta um histórico taxonômico confuso, tendo sido desconsiderado por Hampson (1898) e por Zerny (1912) e Draudt (1915-1917). Hampson (1898) considerou Theages sinônimo de Eucereon Hübner, 1819. É importante observar ainda que diversos pesquisadores da época utilizavam detalhes da venação alar e padrões de coloração como caracteres principais na delimitação dos gêneros dentro de Arctiinae, propondo assim grupos artificiais, visto que em alguns táxons ocorre variação na venação entre as asas de um 
mesmo exemplar (Rego-Barros, 1956; Travassos, 1964). Mais de um século depois da descrição de Theages, Travassos (1964) demonstrou, através do estudo de genitálias e androcônias da espécie-tipo, que o gênero deveria ser revalidado.

Em seu estudo, Travassos (1964) reconheceu outras espécies, que em sua concepção, assemelhavam-se à espécie-tipo do gênero através da observação de caracteres externos. Desse modo, o autor reintegrou a composição de Theages Walker para oito espécies: T. leucophaea, T. flavicaput (Hampson, 1898), T. xantura (Schaus, 1910), T. albidius (Rothschild, 1912), T. griseatum (Rothschild, 1912), T. hoffmanni Travassos, 1962, T. decorum (Schaus, 1910) e T. bricenoi (Rothschild, 1912). É interessante mencionar que todas as espécies inseridas por este autor, com exceção de T. hoffmanni, pertenciam, originalmente, a Eucereon Hübner, 1819.

Travassos (1964) demonstrou ainda a relevância do exame da genitália na caracterização das espécies do gênero, principalmente porque são extremamente semelhantes com relação aos caracteres de asas, abdome e padrões cromáticos, dificultando a análise das variações interespecíficas e intraespecíficas. Contudo, em seu trabalho, Travassos fez apenas uma descrição resumida dos caracteres cromáticos e disponibilizou ilustrações das genitálias masculinas e femininas de apenas seis espécies, deixando lacunas na delimitação do gênero. Ademais, Theages constitui um grupo com informações inexistentes quanto às relações de parentesco entre os seus táxons e sua monofilia nunca foi investigada.

Recentemente, novas contribuições taxonômicas foram propostas para o gênero. Grados \& Mantilla (2018) descreveram três espécies novas para a América do Sul: T. occultus Grados, T. luculentus Grados \& Mantilla e T. pinasi Grados. Contudo, eles descreveram somente a morfologia da genitália masculina desses táxons. Araujo et al., (submetido) conduziram um estudo sobre a morfologia comparada de seis espécies do gênero e sugeriram uma nova combinação para o gênero de uma espécie previamente inserida em Eucereon: T. darantasium (Druce, 1895). Ademais, Theages constitui um grupo com informações inexistentes sobre as relações de parentesco entre seus táxons e sua monofilia nunca foi investigada.

Theages apresenta distribuição neotropical e, do total de espécies atualmente inseridas no gênero, três apresentam como localidade-tipo o Brasil, dentre elas a 
espécie-tipo, T. leucophaea, descrita com base em espécimes coletados no Rio de Janeiro.

\section{9 Órgãos sexuais secundários em Theages}

Os órgãos sexuais secundários, também conhecidos como órgãos androconiais, são comuns em machos de Lepidoptera e comumente localizados no abdome, tórax, pernas ou asas (Birch et al., 1990; Robbins et al., 2012). Diversos lepidópteros utilizam estas estruturas em comportamentos reprodutivos (Egelhaaf et al., 1992). De acordo com Barth (1953), tais órgãos odoríferos são comuns principalmente em lepidópteros com hábito noturno e podem ser estruturalmente diferentes entre espécies distintas.

A maioria das espécies inseridas em Arctiinae apresenta estruturas odoríferas denominadas coremas, em forma de sacos ou tubos cobertos por escamas ou cerdas, geralmente localizadas nos esternitos abdominais VII e VIII (Birch et al., 1990). Tais estruturas são retráteis e encontradas exclusivamente nos machos, estando provavelmente associadas com a cópula (Barth, 1956). Willis \& Birch (1982) demonstraram a presença do comportamento de agregação de indivíduos da espécie Estigmene acrea (Drury, 1773) (Erebidae: Arctinae) ao anoitecer, com diversos machos exibindo seus órgãos odoríferos em um mesmo local, levando à atração das fêmeas e posterior acasalamento.

Acredita-se que a capacidade de eversão do órgão odorífero em Arctiinae é determinada pela inflação pneumática dos tubos, que são virados ao avesso, expondo assim estruturas que liberam hidroxidanaidal, uma substância sintetizada a partir de alcalóides pirrolizidínicos sequestrados pelas larvas ao se alimentarem das plantas hospedeiras (Conner et al., 1981). Grados (2004) mencionou que embora a presença de órgãos odoríferos tenha sido estudada em alguns grupos de lepidópteros, pouco se conhece sobre estas estruturas em representantes de Arctiinae da região neotropical.

Em Theages são encontrados quatro órgãos androconiais dorsais nos tergitos IV a VII do abdome, sendo constituídos por uma fenda transversal, numerosas células glandulares e extremidades com tufos longos de cerdas claras (Travassos, 1964). Contudo, não se conhece detalhes acerca da morfologia e função de tais estruturas odoríferas no gênero. Grados (2004) observou espécimes de T. flavicaput, $T$. 
griseatum, T. xanthura e T. decorum e demonstrou através de uma descrição breve que as escamas dos órgãos androconiais variavam quanto ao tamanho e quantidade entre os tergitos do abdome, porém destacou a importância de novos estudos que poderão fornecer informações sobre de possíveis variações entre as espécies.

De acordo com Simmons et al. (2012), a elucidação da evolução dos órgãos androconiais pode fornecer um passo importante na compreensão de aspectos comportamentais e ecológicos nas linhagens de Arctiinae. Além disso, a caracterização morfológica de tais estruturas poderá viabilizar estudos futuros focados no entendimento dos mecanismos e base química envolvidos nas interações ecológicas do grupo. 


\section{REFERÊNCIAS}

AMORIM, D. S. 2002. Fundamentos de Sistemática Filogenética. Ribeirão Preto, $\mathrm{SP}$, Holos Editora. 154 p.

ARNETT, R. H. JR; SAMUELSON, G. A \& NISHIDA, G. M. 1993. The insect and spider collections of the World. Sandhill Crane Press, Gainesville. 310p.

BÄNZIGER, H. 1982. Fruit-piercing moths in Thailand: a general survey and some new perspectives. Mitteilungen der Schweizerischen Entomologischen Gesellschaft, 55: 213-240.

BARTH, R. 1953. Órgãos odoríferos masculinos de algumas Syntomidae brasileiras (=Ctenuchidae; Lepidoptera). Memorias do Instituto Oswaldo Cruz, 51: 227262.

BARTH, R. 1956. Os tubos abdominais dos machos de alguns Arctiidae e Ctenuchidae do Brasil. Memorias do Instituto Oswaldo Cruz, 54: 499-515.

BARTH. R. 1960. Órgãos odoríferos dos Lepidópteros. Boletim do Parque Nacional do Itatiaia, 7: 1-159.

BECCACECE, H. M.; ZAPATA, A. I.; VILLAFAÑE, N. A. \& DREWNIAK, M. E. 2012. Árctidos nocturnos (Lepidoptera: Erebidae: Arctiinae) del Bosque Serrano de Córdoba, Argentina. Revista de la Sociedad Entomologica Argentina, 71: 99-103.

BEUTEL, R.G. \& POHL, H. 2006. Endopterygote systematics - where do we stand and what is the goal (Hexapoda, Arthropoda)? Systematic Entomology, 31: $202-219$.

BIRCH, M. C. 1972. Male abdominal brush-organs in British Noctuid Moths and their value as a taxonomic character. Entomologist. 105 (1310): 185-205, (1312): 233-244.

BIRCH, M.C.; POPPY, G.M. \& BAKER, T.C. 1990. Scent and eversible scent structures of male moths. Annual Review of Entomology, 35: 25-58. 
BRAZEAU, M. D. 2011. Problematic character coding methods in morphology and their effects. Biological Journal of the Linnean Society, 104: 489-498.

BREMER, K. 1994. Branch support and tree stability. Cladistics, 10: 295-304.

BUTLER, A.G. 1876. On the subfamilies Antichlorinae and Charideinae of the lepidopterous families Zygaenidae and Arctiidae. Journal of the Linnean Society of London (Zoology), 12 (63):408-433. https://doi.org/10.1111/j.10963642.1874.tb01995.x.

CHALWATZIS, N.; HAUF, J.; VAN DE PEER, Y.; KINZELBACH, R. \& ZIMMERMANN, F. K. 1996. 18S ribosomal RNA genes of insects: Primary structure of the genes and molecular phylogeny of Holometabola. Annals Entomological Society of America, 89:775-787.

CONNER, W. E.; EISNER, T.; VANDER MEER, R. K.; GUERRERO, A. \& MEINWALD, J. 1981. Precopulatory sexual interaction in an arctiid moth (Utetheisa ornatrix): role of pheromone derived from alkaloids. Behavioral Ecology and Sociobiology, 9: 277-235.

CROWSON, R.A.; ROLFE, W.D.I.; SMART, J.; WATERSTON, C.D.; WILLEY, E.C. \& WOOTTON, R.J. 1967. In: The Fossil Record, Arthropoda. London, Geological Society. p. 499-534.

CRUMB, S. E. 1934. A classification of some noctuid larvae of the subfamily Hypeninae. Entomologica Americana, 14: 133-196.

De JONG, R. 1982. Secondary sexual characters in Celaenorrhinus and the delimitation of the genus (Lepidoptera: Hesperiidae). Jounal of Natural History, 16, 695-705.

De PINNA, M. C. 1991. Concepts and tests of homology in the cladistics paradigm. Cladistics, 7: 367-394.

DESCHAMPS. B. 1835. Recherches microscopiques sur l'organisation des ailes des Lépidoptères. Annales des Sciences Naturelles, 3: 300-304. 
DETHIER, V. G. 1941. The antennae of lepidopterous larvae. Bulletin of the Museum of Comparative Zoology, 87: 455-507.

DEVRIES, P. J. (1986). Hostplant Records and Natural History Notes on Costa Rican Butterflies (Papilionidae, Pieridae e Nymphalidae). The Journal of Research on the Lepidoptera, 24: 290- 333.

DINIZ, I. R.; MORAIS, H. C.; BOTELHO, A. M. F.; VENTUROLI, F. \& CABRAL, B. C. 1999. Lepidopteran Caterpillar fauna on Lactiferous host plants in the Central Brazilian Cerrado. Revista Brasileira de Biologia, 59: 627-635.

DRAUDT, M. 1915-1917. Family Syntomidae. In: Seitz, A. (Ed.). The Macrolepidoptera of the world. Sttutgart, A. Kernen. v. 6, p. 33- 230.

DRUCE, H. 1891-1900. Biología Centrali-Americana. Insecta. LepidopteraHeterocera. London, R.H. Porter. v. 2, 622p. + 101pl.

DUARTE, M.; MARCONATO, G.; SPECHT, A. \& CASAGRANDE, M. M. 2012. Lepidoptera. In: Rafael, J.A.; Melo; G.A.R.; Carvalho; C.J.B.; Casari, S. A. \& Constantino, R. (Eds.). Insetos do Brasil. Diversidade e Taxonomia. Ribeirão Preto, SP, Holos Editora. p. 626-682.

EGELHAAF, A.; RICK-WAGNER, S. \& SCHNEIDER, D. 1992. Development of the male scent organ of Creatonotos transiens (Lepidoptera, Arctiidae) during metamorphosis. Zoomorphology, 111: 125-139.

ELDIJK, T. J. B.; WAPPLER, T.; STROTHER, P. K.; WIHST, C. M. H.; RAJAEI, H.; VISSCHE, H.; SCHOOTBRUGEL, B. 2018. A Triassic-Jurassic window into the evolution of Lepidoptera. Sciences advances, v 4: e1701568, p. 1-7.

FIBIGER, M. \& LAFONTAINE, J. D. 2005. A review of the higher classification of the Noctuoidea (Lepidoptera) with special reference to the holarctic fauna. Esperiana, 11: 7-92.

FITCH, W. 1971. Toward defining the course of evolution: minimal change for a specific tree topology. Systematic Zoology, 20: 406-416. 
FORBES, A. A.; BAGLEY, R. K.; BEER, M. A.; HIPPEE, A. C. \& WIDMAYER, H. A. 2018. Quantifying the unquantifable: why Hymenoptera, not Coleoptera, is the most speciose animal order. BMC Ecology, 18(21): 1-11.

FORBES, W. T. M. 1910. A structural study of some caterpillars. Annals of the Entomological Society of America, 3: 94-143.

FRACKER, S. B. 1915. The classification of lepidopterous larvae. Illinois Biological Monographs, 2: 1-170.

GASTON, K. J. 1991. The magnitude of global insect species richness. Conservation Biology, 5(3): 283- 296.

GOLDSTEIN, P.Z. 2017. Diversity and significance of Lepidoptera: a phylogenetic perspective. In: Foottit, R.G. \& Adler, P.H. (Eds.). Insect biodiversity: science and society. 2.ed. New Jersey, John Wiley \& Sons Ltd. v. 1, p. 497498.

GOLOBOFF, P; FARRIS, P. \& NIXON, K. 2008. Tree analysis using New Technology. Disponível em: <http://www.cladistics.com/aboutTNT.html $>$ (acesso em 27 maio 2018).

GRADOS, J. \& MANTILHA, K. 2018. Tres nuevas especies del género Theages Walker, 1855 (Lepidoptera: Erebidae: Arctiini) de Perú y Ecuador. Revista Peruana de Biologia, 25(1):11 - 22 .

GRADOS, J. 2002. Los Arctiidae y Sphingidae (Lepidoptera: Heterocera) del santuario histórico de Machu Picchu, Cuzco, Perú: estudio preliminar. Revista Peruana de Biologia, 9: $16-22$.

GRADOS, J. 2004. Órganos androconiales abdominales peculiares en Theages Walker, 1855 (Lepidoptera: Arctiidae). Revista Peruana de Biologia, 11: 27 30.

GRADOS, J.; ESPINOZA, C.; RAMÍREZ, J.J. \& CENTENO, P. 2013. Siete nuevos registros de Arctiini (Arctiinae: Erebidae: Lepidoptera) para Perú. Revista Peruana de Biologia, 20:171- 176. 
GRIMALDI, D.; ENGEL, M. S. 2005. Evolution of the insects. New York, Cambridge University Press. 755p.

HAMPSON, G. F. 1898-1913. Catalogue of the Lepidoptera Phalaenae. In: Catalogue of the Arctiadae (Nolinae, Lithosianae in the Collection of the British Museum. London, Printed by Order of Trustees. p. 1-13.

HAMPSON, G. F. 1898. Catalogue of the Syntomidae in the collection of the British Museum. In: Catalogue of the Lepidoptera Phalenae. London, Printed by Order of Trustees. $559 \mathrm{pp}$.

HAMPSON, G.F. (1914). Catalogue of the Amatidae and Arctiadae (Nolinae and Lithosianae) in the collection of the British Museum. In: Catalogue of the Lepidoptera Phalenae in the British Museum. London, Trustees of the British Museum. Supplemento 1, 858 p.

HAMPSON, G.F. 1915. Catalogue of the Lepidoptera Phalaenae in the British Museum. London, Printed by the order of the Trustees. Supplement I, Plates IXLI, 71p.

HAWKINGS, J. A.; HUGleS, C. E. \& SCOTLAND, R. W. 1997. Primary Homology Assessment, Characters and Character States. Cladistics, 13: 275283.

HENNIG, W. 1966. Phylogenetic Systematics. Urbana, Illinois University Press.

HEPPNER, J. B. (1991). Faunal regions and the diversity of Lepidoptera. Tropical Lepidoptera, 2(Suppl. 1):1-85.

HEPPNER, J.B. 1998. Classification of Lepidoptera. Part 1. Introduction. Holarctic Lepidoptera, 5 (Suppl. 1) : 1-148.

HERNÁNDEZ-ROLDÁN, J. L.; BOFILL, R.; DAPPORTO, L.; MUNGUIRA, M. L.; VILA, ROGER. 2014. Morphological and chemical analysis of male scent organs in the butterfly genus Pyrgus (Lepidoptera: Hesperiidae). Organisms diversity and evolution, v. 14, p. 269-278.

HOFFMANN, F. 1936. Beiträge zur Lepidopterenfauna von Santa Catharina. 
Entomologische Rundschau, 53:46-48.

HOLLOWAY, J.D. 2011. The Moths of Borneo (part 2): families Phaudidae, Himantopteridae and Zygaenidae; revised and annotated checklist. Malayan Nature Journal, 63:1-548.

JACOBSON, N. L. \& WELLER, S. J. 2002. A cladistic study of the Arctiidae (Lepidoptera) by using characters of immatures and adults. Texas, Entomological Society of America. Thomas Say Publications in Entomology, Monographs. 97p.

KIRBY, W. F. 1892. A synonymic catalogue of Lepidoptera Heterocera (moths). Volume 1. Sphinges and Bombyces. London, Gurney \& Jackson. 951p.

KITCHING, I. J. \& RAWLINGS, J. E. 1998. The Noctuoidea. In: Kristensen, N. P. (Ed.). Arthropoda: Insecta. Lepidoptera, Moths and Butterflies: evolution, systematic and biogeography, v. 1. Edited by Fischer, M. (Ed.). Handbuch der Zoologie/Handbook of Zoology. Berlin, Walter de Gruyter Band. v. IV, p. 389-394.

KITCHING, I. J. 1984. An historical review of the higher classification of the Noctuidae (Lepidoptera). Bulletin of the British Museum (Natural History): Entomology, 49:153-234.

KITCHING, I. J.; FOREY, P. L.; HUMPHRIES, C. J.; WILLIAMS, D. M. (1998). Cladistics: the theory and practice of parsimony analysis. 2.ed. Oxford University Press.

KJER, K. M.; BLAHNIK, R. J. \& HOLZENTHAL, R. W. 2001. Phylogeny of Trichoptera (Caddisflies): characterization of signal and noise within multiple Datasets. Systematic Biology, 50 (6): 781-816.

KLOTS A. (1970). Lepidoptera. In: Tuxen, S.L. (Ed.). Taxonomist's glossary of genitalia in insects. Copenhagen, Munksgaard. p. 88-111. 
KRISTENSEN, N. P; SCOBLE, M. J. \& KARSHOLT, O. 2007. Lepidoptera phylogeny and systematics: the state of inventoring moth and butterfly diversity. Zootaxa, 1668: 699-747.

KRISTENSEN, N. P. 1997. Early evolution of the Lepidoptera C Trichoptera lineage: Phylogeny and the evolutionary scenario. Mémoires du Museum National d' Histoire naturelle nouvelle serie. Série A Zoologie, 173:253-271.

KRISTENSEN, N.P. \& SKALSKI, A.W. 1998. Phylogeny and palaeontology. In: Kristensen, N. P. (Ed.). Arthropoda: Insecta. Lepidoptera, Moths and Butterflies: evolution, systematic and biogeography, v. 1. Edited by Fischer, M. (Ed.). Handbuch der Zoologie/Handbook of Zoology. Berlin, Walter de Gruyter. Band. v. IV, p. 7-25.

KRISTENSEN, N.P. 1999. Phylogeny of endopterygote insects, the most successful lineage of living organisms. European Journal of Entomology, 96: 237-253.

LAFONTAINE, J. D. \& FIBIGER, M. (2006). Revised higher classification of the Noctuoidea (Lepidoptera). Canadian Entomologist, 138: 610-635.

LAFONTAINE, J. D. \& SCHMIDT, B. C. 2010. Annotated check list of the Noctuoidea (Insecta, Lepidoptera) of North America north of Mexico. ZooKeys, 40: 1-239.

LAMAS G.; GRADOS, J. 1996. Sinopsis de los Pericopinae (Lepidoptera: Arctiidae) del Perú, con comentarios taxonómicos y la descripción de una nueva subespecie. Revista Peruana de Entomologia, 39: 21-28.

LAMAS, G. 2008. La sistemática sobre mariposas (Lepidoptera: Hesperioidea y Papilionoidea) en el mundo: estado actual y perpectivas futures. In: Bousquets, J.L. \& Lanteri, A. (Org). Contribuiciones taxonómicas en ordenes de insetos hipererdiversos. Mexico, UNAM. p.57-70. (III Reunión Annual de la Red Iberoamericana de Biogeografía y Entomologia Systematica)

MA, P. W. K. \& RAMASWAMY, S. B. 2003. Biology and ultrastructure of sex pheromone-producing tissue. In: Blomquist, G. J. \& Vogt, R. G. Insect pheromone biochemistry and molecular biology: the biosynthesis and 
detection of pheromones and plant volatiles. New York, Academic Press. p. $18-51$.

MADDISON, W. P. \& MADDISON, D. R. 2008. Mesquite: a modular system for evolutionary analysis. Version 2.01. Em: $<\mathrm{http}: / /$ mesquiteproject.org>, 2008.

MADDISON, W.P. \& DONOGHUE, M.; MADDISON, D.R. (1984). Outgroup analysis and parsimony. Systematic Zoology, 33: 83-103.

MILLER, J. S. 1991. Cladistics and classification of the Notodontidae (Lepidoptera, Noctuoidea) based on larval and adult morphology. Bulletin of the American Museum of Natural History, 204:1-230.

MIRANDE, J.M. (2009). Weighted parsimony phylogeny of the family Characidae (Teleostei: Characiformes). Cladistics, 25: 574-613.

Mitchell, A.; CHO, S.; RegieR, J. C.; MitTeR, C.; POOLE, R. W. \& MATTHEWS, M. 1997. Phylogenetic utility of elongation factor1 alpha in Noctuoidea (Insecta: Lepidoptera): the limits of synonymous substitution. Molecular Biology and Evolution, 14:381-390.

MITCHELL, A.; MITTER, C. \& REGIER, J. C. 2000. More taxa or more characters revisited: combining data from nuclear protein encoding genes for phylogenetic analyses of Noctuoidea (Insecta: Lepidoptera). Systematic Biology, 49: 202224.

MITCHELL, A.; MITTER, C. \& REGIER, J.C. 2006. Systematics and evolution of the cutworm moths (Lepidoptera: Noctuidae): evidence from two proteincoding nuclear genes. Systematic Entomology, 31: 21-46.

MITTER, C.; DAVIS, D.R. \& CUMMINGS, M.P. 2017. Phylogeny and evolution of Lepidoptera. Annual Review of Entomology, 62: 265-283.

MORAES, S. S. \& DUARTE, M. 2009. Morfologia externa comparada das três espécies do complexo Telchin licus (Drury) (Lepidoptera, Castiinidae) com uma sinonímia. Revista Brasileira de Entomologia, 53:245-265. 
MOSHER, E. 1916. A classification of the Lepidoptera based on characters of the pupa. Bulletin of the Illinois State Laboratory of Natural History, 12: 17159.

MUTANEN, M.; WAHLBERG, N. \& KAILA, L. 2010. Comprehensive gene and taxon coverage elucidates radiation patterns in moths and butterflies. Proceedings of the Royal Society of London, (B), 277: 2839-2848.

NEW, T. R. 1997. Are Lepidoptera an effective "umbrella group" for biodiversity conservation? Journal of Insect Conservation, 1:5- 12.

NIXON, C. K.; CARPENTER, J, M. 1993. On outgroups. Cladistics, 9: 413-426.

NIXON, K. C. WinClada. Disponível em: http://www.cladistics.com/aboutWinc.htm acesso em 27 maio de 2018.

PARRA, L. E.; JIMÉNEZ-URRUTIA, M. C. \& ZAMORA-MANZUR, C. 2009. Revision of the genus Hoplosauris Butler 1882 (Lepidoptera: Geometridae). Zootaxa 1989: 39-54.

PEÑA, C. \& LAMAS, G. 2005. Revision of the butterfly genus Forsterinaria Gray, 1973 (Lepidoptera: Nymphalidae, Satyrinae). Revista Peruana de Biología 12(1): 5-48.

PINHEIRO, L. R. \& DUARTE, M. 2013. Taxonomic notes on Ctenuchina, Euchromiina, and Phaegopterina (Lepidoptera, Erebidae, Arctiinae, Arctiini). Florida Entomologist, 96:351-359.

PIOVESAN, M.; ORLANDIN, E.; FAVRETTO, M. A. \& SANTOS, E. B. 2014. Contribuição para o conhecimento da lepidopterofauna de Santa Catarina, Brasil. Scientia Plena, 10:1- 32.

PLATINICK, N. I.; GRISWOLD, C. E.; CODDINGTON, J. A. 1991. On missing entries in cladistics analysis. Cladistics, 7:337-343.

POWELL, J. A.; MITTER, C. \& FARREL, B. 1998. Evolution of food preferences in Lepidoptera. In: Kristensen, N. P. (Ed.). Arthropoda: Insecta. Lepidoptera, Moths and Butterflies: evolution, systematic and biogeography, v. 1. Edited by 
Fischer, M. (Ed.). Handbuch der Zoologie/Handbook of Zoology. Berlin, Walter de Gruyter. Band. v. IV, p. 403-422.

RAFAEL, J. A.; MELO G. A. R. \& CARVALHO, J. B. 2012. Insetos do Brasil: Diversidade e Taxonomia. Ribeirão Preto, SP, Holos Editora. 810p.

REGieR, J. C.; MITTER, C.; MITTER, K.; CUMMINGS, M. P.; BAZINET, A. L.; HALLWACH, W.; JANZEN, D. H.; ZWICK, A. (2016). Further progress on the phylogeny of Noctuoidea (Insecta: Lepidoptera) using an expanded gene sample. Systematic Entomology, 1-12, DOI: 10.1111/syen.12199.

REGIER, J.C.; ZWICK, A. \& CUMMINGS, M.P. 2009. Toward reconstructing the evolution of advanced moths and butterflies (Lepidoptera: Ditrysia): an initial molecular study. BMC Evolutionary Biology, 9:280-300.

REGO-BARROS, A. R. 1956. Contribuição ao estudo da família Arctiidae IV. Rediagnose de Hemihylaea Hampson 1901 e descrição de novos gêneros (Lepidoptera-Heterocera). Boletim do. Museu Nacional, Zoologia, 140:1-65.

REIS, R. E. 2008. Inferência filogenética: suporte. Disponível em: $<$ http://www.pucrs.br/fabio/reis/suporte.pdf $>$. Acesso em 27 jul. 2018.

RIPLEY, L. B. 1923. The external morphology and postembryology of noctuid larvae. Illinois Biological Monographs, 8: 1-102.

ROBBINS, R, K.; MARTINS, A. R. P.; BUSBY, R. C. \& DUARTE, M. 2012. Loss of male secondary sexual structures in allopatry in the Neotropical butterfly genus Arcas (Lycaenidae: Theclinae: Eumaeini). Insect Systematics \& Evolution, 43: 35-65.

ROBBINS, R. K. \& HENSON, P. M. 1986. Why Pieris rapae is a better name than Artogeia rapae (Pieridae). Journal of the Lepidopterists' Society 40(2): 79-92.

ROBINSON, G.S. 1976. The preparation of slides of Lepidoptera genitalia with special reference to the Microlepidoptera. Entomologist's Gazette, 27:127132. 
ROSS, H. H. 1967. The evolution and the past dispersal of the Trichoptera. Annual Review of Entomology, 12: 169-206.

ROTHSCHILD, W. 1912. New Syntomidae. Novitates Zoologicae, 19 (2):151-186.

SCHAUS, W. 1910. Descriptions of new Heterocera from Costa Rica. Annals and Magazine of Natural History, 8 (6): 189-211.

SCHNEIDER D.; LEGAL, L.; DIERL, W. \& WINK, M. (1999). Androconial hairbrushes of the Syntomis (Amata) phegea (L.) group (Lepidoptera: Ctenuchinae): a synapomorphic character supported by sequence data of the mitochondrial 16S rRNA gene. Zeitschrift für Naturforschung, 54: 11191139.

SCHUH, R. T. 2000. Biological systematics: principals and applications. New York, Cornell University Press.

SCOBLE, M.J. 1992. The Lepidoptera: form, function and diversity. Oxford, Oxford University Press.

SCOTLAND, R. E. \& PENNINGTON, R. T. 2000. Homology and Systematics: coding characters for phylogenetic analysis. London, Taylor \& Francis. 217p. (The Systematic Association Special Volume Series 58).

SERENO P. C. 2007. Logical basis for morphological characters in phylogenetics. Cladistics, 23: 565-587.

SIBATANI, A.; OGATA, M.; OKADA, Y. \& OKAGAKI, H. 1954. Male genitalia of Lepidoptera: morphology and nomenclature. I. Divisions of the valvae in Rophalocera, Phalaenidae (Noctuidae) and Geometridae. Annals of the Entomological Society of America, 47: 93-106.

SIMMONS, R. B. \& WELLER, S. J. 2001. Utility and evolution of cytochrome b in insects. Molecular Phylogenetics and Evolution, 20: 196-210.

SIMMONS, R. B.; WELLER, S. J. \& JOHNSON, S. J. 2012. The evolution of androconia in mimetic tiger moths (Noctudoidea: Erebidae: Arctiinae: Ctenuchina and Euchromiina). Annals Entomological Society of America, 
105(6): 804-816.

SIMMONS, R.B. \& WELLER, S.J. 2006. Review of the Sphecosoma genus group using adult morphology (Lepidoptera: Arctiidae). Texas, Entomological Society of America. 108p. (Thomas Say Publications in Entomology: Monographs)

STRAND, E. 1920. Kritische Bemerkungen und Berichtigungen zum Supplementband I des Hampson'schen "Catalogue of the Lepidoptera Phalaenae". Deutsche Entomologische Zeitschrift Iris, 34(3/4):217-226.

TRAVASSOS, L. (1962). Gênero Theages Walker, 1855 (Lepidoptera) Nota preliminar. Atas Sociedade Biologica, Rio de Janeiro. p. 58-59.

TRAVASSOS, L. (1964). Contribuição ao conhecimento dos Arctiidae (LepidopteraHeterocera) Gênero Theages Walker, 1855. Memorias do Instituto Oswaldo Cruz, Rio de Janeiro, 36: p. 173- 188.

TRAVASSOS, L. 1952. Contribuição ao conhecimento dos "Arctiidae". XXVIII. Lepidoptera, Heterocera. Revista Brasileira de Biologia, 12(2): 151-160.

TRAVASSOS, L. P. 1959. Contribuição ao conhecimento dos Arctiidae. XLII. Gênero Eucereon Hubner 1819 (Lepidoptera, Heterocera). Memorias do Instituto Oswaldo Cruz, 57 (2): 171-190.

VAN NIEUKERKEN, E.J.; KAILA, L.; KITCHING, I.J.; KRISTENSEN, N.P.; LEES, D.C.; MINET, J.; MITTER, C.; MUTANEN, M.; REGIER, J.C.; SIMONSEN, T.J.; WAHLBERG, N.; YEN, S.H.; ZAHIRI, R.; ADAMSKI, D.; BAIXERAS, J.; BARTSCH, D.; BENGTSSON, B. A.; BROWN, J. W.; BUCHELI, S. R.; DAVIS, D. R.; DE PRINS, J.; DE PRINS, W.; EPSTEIN, M. E.; GENTILI-POOLE, P.; GIELIS, C.; HÄTTENSCHWILER, P.; HAUSMANN, A.; HOLLOWAY, J. D.; KALLIES, A.; KARSHOLT, O.; KAWAHARA, A. Y.; KOSTER, S.; KOZLOV, M. V.; LAFONTAINE, D.; LAMAS, G.; LANDRY, J. F.; LEE, S. M. N.; PARK, K. T.; PENZ, C.; ROTA, J.; SCHINTLMEISTER, A.; SCHMIDT, B. C.; SOHN, J. C.; SOLIS, M. A.; TARMANN, G. M.; WARREN, A. D.; WELLER, S.; YAKOVLEV, R. V.; ZOLOTUHIN, V. V.; ZWICK, A. (2011). Order Lepidoptera Linnaeus, 1758. 
In: Zhang, Z. Q. (Ed.). Animal biodiversity: 42 An outline of higher-level classification and survey of taxonomic richness. Zootaxa, 3148: 212-221.

WALKER, F. 1855. List of specimens of lepidopterous insects in the collections of the British Museum. London, Printed by Order of the Trustees. v. 3, 278p.

WATROUS, L.; WHEELER, Q. 1981. The out-group comparison method of character analysis. Systematic Zoology, 30: 1-11.

WElleR, S; Da COSTA, M.; SIMMONS, R.; DITTMAR, K. \& WHITING, M. 2009. Evolution and taxonomic confusion in Arctiidae. In: Conner, W. E. (Ed.). Tiger moths and woolly bears: behavior, ecology and evolution of the Arctiidae. New York, Oxford University Press. p. 11-30.

WELLER, S. J.; JACOBSON, N. \& CONNER, W. E. 1999. The evolution of chemical defenses and mating systems in tiger moths (Lepidoptera: Arctiidae). Biological Journal of the Linnean Society, 68: 557-578.

WELLER, S.J.; PASHLEY, D.P.; MARTIN, J.A. \& CONSTABLE, J.L. 1994. Phylogeny of noctuoid moths and the utility of combining independent nuclear and mitochondrial genes. Systematic Biology, 43:194-211.

WHALLEY, P. 1978. New taxa of fossil and recent Micropterigidae wirh a discussion of their evolution and a comment on the evolution of the Lepidoptera (Insecta). Annals of the Transvaal Museum, 31: 72-86.

WILLIS, M. A.; BIRCH, M. C. 1982. Male lek formation and female calling in a population of the arctiid moth, Estigmene acrea. Science, 218:168-70.

WINTER, W. D. 2000. Basic techniques for observing and studying moths and butterflies. Memoirs of the Lepidopterists' Society, 5:1- 444.

ZAHIRI, R.; HOLLOWAY, J. D.; KITCHING, I. J.; LAFONTAINE, J.; MUTANEN, M. \& WAHLBERG, N. 2011. Molecular phylogenetics of Erebidae (Lepidoptera, Noctuoidea). Systematic Entomology, 37: 102-124. 
ZAHIRI, R.; KITCHING, I.J.; LAFONTAINE, J.D.; MUTANEN, M.; KAILA, L.; HOLLOWAY, J.D. \& WAHLBERG, N. 2010. A new molecular phylogeny offers hope for a stable family-level classification of the Noctuoidea (Lepidoptera). Zoologica Scripta, 40:158-173.

ZASPEL, J. M.; WELLER, S. J.; WARDWELL, C. T.; ZAHIRI, R.; WAHLBERG, N. 2014. Phylogeny and evolution of pharmacophagy in Tiger Moths (Lepidoptera: Erebidae: Arctiinae). Plos One, 9(7):e101975.

ZENKER, M. M.; WAHLBERG, N.; BREHM, G.; TESTON, J. A.; PRZYBYLOWICZ, L.; PIE, M. R. \& FREITAS, A. V. L. 2016. Systematics and origin of moths in the subfamily Arctiinae (Lepidoptera, Erebidae) in the Neotropical region. Zoologica Scripta, 46: 348-362.

ZERNY, H. 1912. Syntomidae. In: Wagner, H. (Ed.). Lepidopterorum Catalogus, Pars 7. Berlin, W. Junk. p. 179-179. https://doi.org/10.5962/bhl.title.124036.

ZERNY, H. 1931. Beiträge zur Kenntnis der Syntomiden. Deutsche Entomologische Zeitschrift, Iris, 45: 1-27.

ZHANG, B.C. 1994. Index of Economically Important Lepidoptera. Wallingford, Conn., CAB International. 